\title{
Effect of noise in blending and deblending
}

\author{
Guus Berkhout ${ }^{1}$ and Gerrit Blacquière ${ }^{1}$
}

\begin{abstract}
If simultaneous shooting is carried out by incoherent source arrays, being the condition of blended acquisition, the deblending process generates shot records with a very low residual interference (blending noise). We found, theoretically and numerically, that deblended shot records had a better background-related signal-to-noise ratio than shot records in unblended surveys. This improvement increased with increasing blending fold and decreasing survey time. An interesting consequence of this property is that blended surveys can be carried out under more severe noise conditions than unblended surveys. It is advisable to optimize the survey time in areas with a large background noise level or in areas with severe environmental restrictions.
\end{abstract}

\section{INTRODUCTION}

Unlike in traditional seismic acquisition, in blended acquisition, temporal overlap between the shot records is allowed. This enables densely sampled, wide-azimuth geometries with favorable economics. In land seismics, the concept of interfering shot records is already known from the use of orthogonal signals in vibroseis acquisition; see Bagaini (2006) for an overview of the various methods used in the industry. For the marine case, in which impulsive sources are used, Beasley et al. (1998) propose to fire such sources simultaneously with large distances between them such that CMP processing already results in good separation. Stefani et al. (2007) further develop this concept and introduce small random time delays as well: jittered simultaneous shooting. The reader is also referred to Vaage (2002), Ikelle (2007), Hampson et al. (2008), and Howe et al. (2008).

The concept of blending in acquisition and processing is introduced by Berkhout (2008). Blended acquisition is the continuous recording of incoherent, multisource responses that overlap in time ("blended shot records"). The multisource array properties are determined by the combination of offsets, azimuths, source strengths, and source delay times. Advanced encoding of source signatures, e.g., when using seismic land or marine vibrators, is optional. The distances between the involved sources, as well as the firing time intervals, may vary from small to large. An essential requirement for a blended source array is its incoherent, multiwavefront property, leading to the alternative terminology "incoherent shooting" (Berkhout et al., 2012). This condition excludes the situation of coherent wavefronts such as plane waves. Bear in mind that seismic blending (SB) is fundamentally different from seismic interferometry (SI): Unlike in SI, in SB, the position, signal property, and blending code are known for each individual source.

A seismic shot record, either blended or unblended, contains the earth response of planned, man-made sources as well as signals from other sources such as industrial activities, traffic, wind, and in the marine case also flow noise, breaking waves, etc. In addition, such a record contains noise that is related to the acquisition equipment, e.g., electronic noise, quantization noise, etc. The recorded events that are not related to the planned source(s) are referred to as the background noise (source-generated noise is considered as signal). We will assume that the background noise is "stationary," meaning that its power is time invariant during the survey.

One of the valuable properties of SB is that in a blended survey, more sources can be deployed within a certain survey time than in the corresponding regular, unblended survey. This is because it is no longer necessary to wait for all reflections to be recorded before firing the next source. In the seismic literature (see e.g., Pecholcs et al., 2010; Abma et al., 2012; Berkhout et al., 2012; Krupovnickas et al., 2012), this property - keeping the survey time within limits - is considered to be a benefit related to economics. Beasley et al. (2012), however, suspect that the observed higher resolution in their field results is not only due to the improved source sampling characteristics of blended acquisition. They argue that the signal-tonoise ratio $(\mathrm{S} / \mathrm{N})$ of blended data is likely to be higher than that of traditionally acquired data, and they speculate that this property of blending may explain why their improvements are beyond expectation. Berkhout and Blacquière (2012) conclude that the

Manuscript received by the Editor 11 March 2013; revised manuscript received 4 May 2013; published online 8 August 2013.

${ }^{1}$ Delft University of Technology, Faculty of Civil Engineering and Geosciences, Delft, The Netherlands. E-mail: a.j.berkhout@tudelft.nl; g.blacquiere@ tudelft.nl.

(C) 2013 Society of Exploration Geophysicists. All rights reserved. 
signal-to-background-noise ratio of a field-blended survey must be higher than of a comparable traditional survey. This is because the power of the signal (total signal energy divided by the effective survey time) increases in blended acquisition, not only if the number of sources increases, but also if the survey time decreases. On the other hand, the power of the background noise is independent of whatever we do in the blending process. Hence, a shorter recording time not only favors economics, it also favors quality, particularly in areas with a high background noise level. Of course, this important conclusion is only valid if the incoherency condition of the blended source array is fulfilled.

In this Letter, our conclusion is explained by a theoretical analysis, and the results are illustrated by numerical examples.

\section{S/N IN BLENDED DATA}

Let us consider the situation of the responses of $M$ sources that are either recorded individually, leading to $M$ unblended shot records, or that are recorded in a blended fashion, leading to a single blended shot record.

Individually acquired shot records can be formulated as (Berkhout, 1982)

$$
\mathbf{P}_{k}=\mathbf{D X S} \mathbf{S}_{k}+\mathbf{N}_{k} \quad \text { for } k=1, \ldots, M .
$$

In equation 1 matrix $\mathbf{X}$ is the earth's transfer operator that includes the interaction with the surface. Source vector $\mathbf{S}_{k}$ represents source (array) $k$, generating a downgoing source wavefield. The source wavelet may be impulsive, as in the case of an air gun or a dynamite source, or it may be nonimpulsive, as in the case of a seismic land or marine vibrator. In detector matrix $\mathbf{D}$, each row represents a receiver (array), generating one seismic trace. The response of source $k$ is given by data vector $\mathbf{P}_{k}$. It includes background noise realization $\mathbf{N}_{k}$. We consider noise realizations in the different shot records to be uncorrelated. In the frequency domain, equation 1 refers to one frequency component.

When acquired in a blended way, i.e., field blending, the obtained blended shot record is given by (Berkhout, 2008)

$$
\mathbf{P}_{j}^{\prime}=\mathbf{D X S} \boldsymbol{\Gamma}_{j}+\mathbf{N}_{j}
$$

or

$$
\mathbf{P}_{j}^{\prime}=\mathbf{D X} \sum_{k=1}^{M} \mathbf{S}_{k} \gamma_{k j}+\mathbf{N}_{j}
$$

where superscript $/$ indicates blending, $\mathbf{N}_{j}$ equals the background noise in blended field record $\mathbf{P}_{j}^{\prime}$, and $\gamma_{k j}$ represents the blending code (being some phase operator). For instance, if time delays $\tau_{k j}$ are applied, we may write $\gamma_{k j}=\exp \left(-j \omega \tau_{k j}\right)$. Note that the power of noise realization $\mathbf{N}_{j}$ in equations $2 \mathrm{a}$ and $2 \mathrm{~b}$ equals the power of noise realization $\mathbf{N}_{k}$ in equation 1 .
Figure 1. Comparison between (a) computer blending and (b) field blending, using a blended array with five sources. As expected, the $\mathrm{S} / \mathrm{N}$ of the field-blended data is a factor of $\sqrt{5}$ better than of the computer-blended data.

Figure 2. Comparison between (a) unblended field data and (b) the result of deblending fieldblended data, using again a blended array with five sources. As expected, the $\mathrm{S} / \mathrm{N}$ of the deblended field data is a factor of $\sqrt{5}$ better than of the unblended field data.

Figure 3. Comparison between (a) unblended field data and (b) the result of deblending computer-blended data, using again a blended array with five sources. As expected, the $\mathrm{S} / \mathrm{N}$ is the same in the two cases. a)

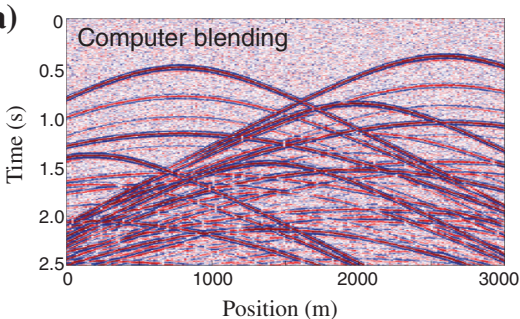

a)

b)

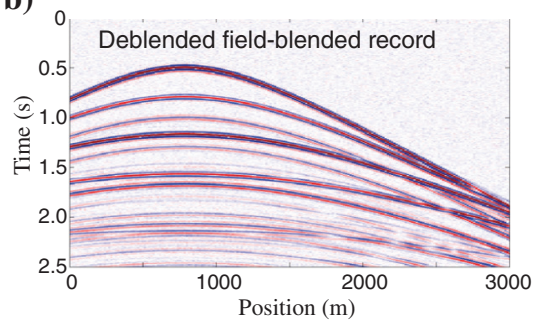

b)

a)
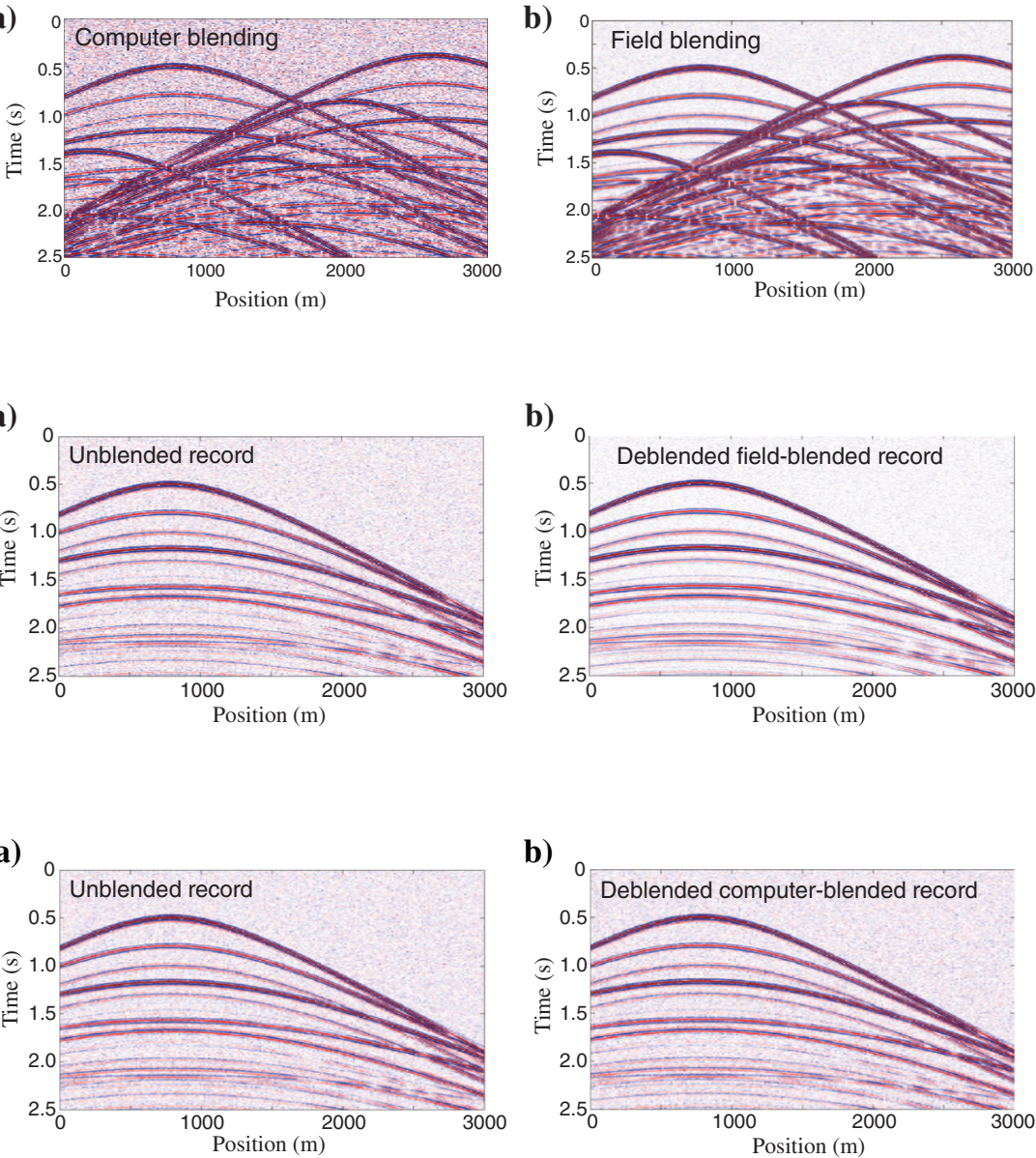

b)

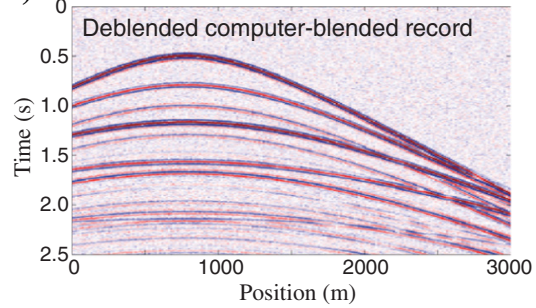

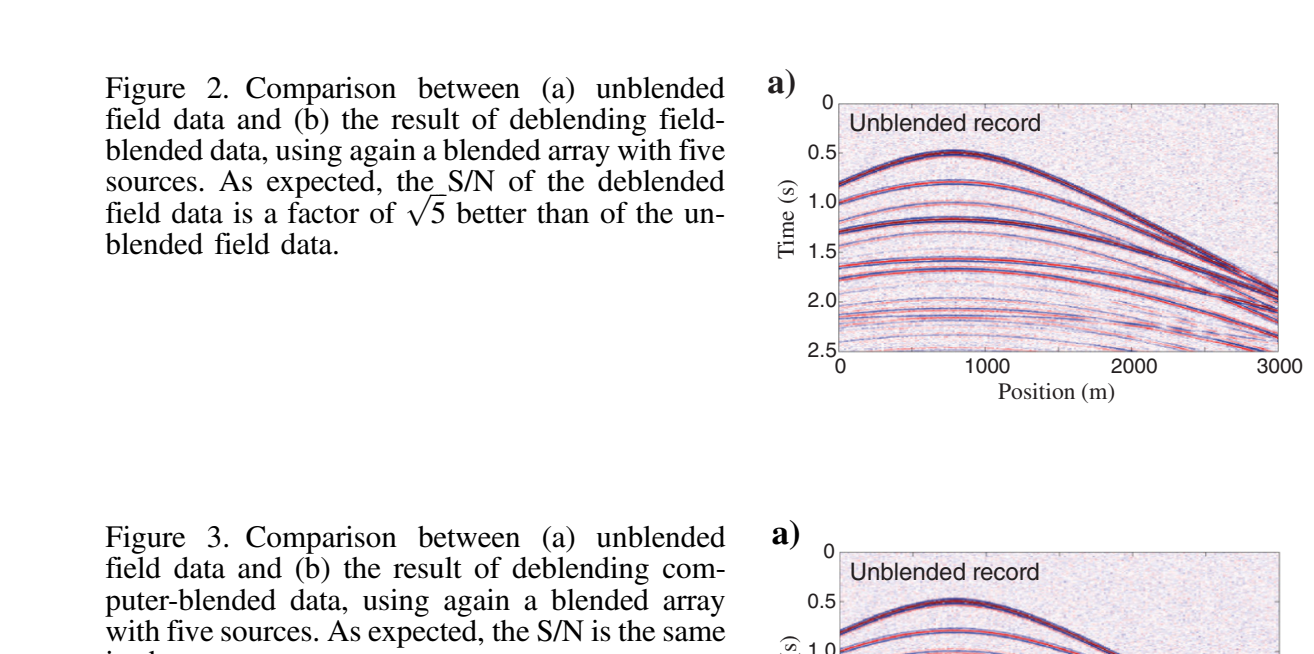


We start with the situation that the survey duration $T^{\prime}$ of the blended survey is the same as the survey duration $T$ of the unblended survey. Here survey duration should be interpreted as "effective," meaning that it corresponds to the total recording time. For the equal duration situation $\left(T^{\prime}=T\right)$, the blending performance indicator (BPI) is given by (Berkhout, 2008)

$$
\mathrm{BPI}=\left(N_{s}^{\prime} / N_{s}\right) \times\left(T / T^{\prime}\right)=N_{s}^{\prime} / N_{s},
$$

where $N_{s}^{\prime}$ equals the number of sources in the blended survey and $N_{s}$ equals the number of sources in the unblended survey. We refer to the ratio $N_{s}^{\prime} / N_{s}$ as the blending fold. If all blended source arrays contain the same number of sources $(M)$, then $N_{s}^{\prime} / N_{s}=M$.

Apart from blending in the field (physical blending), we can also blend unblended field records in the computer (numerical blending):

$$
\mathbf{P}_{j}^{\prime}=\mathbf{P \Gamma}_{j},
$$

or, substituting equation 1 ,

$$
\mathbf{P}_{j}^{\prime}=\mathbf{D X} \sum_{k=1}^{M} \mathbf{S}_{k} \gamma_{k j}+\sum_{k=1}^{M} \mathbf{N}_{k} \gamma_{k j},
$$

where we assume that the power of $\mathbf{N}_{k} \gamma_{k j}$ equals the power of $\mathbf{N}_{k}$ $\left(\gamma_{k j}\right.$ is a phase operator). If we now compare the data set that was blended in the field (equation $2 \mathrm{~b}$ ) with the one blended in the computer (equation $4 \mathrm{~b}$ ), it becomes clear that the latter contains more noise. In the case of computer blending, the noise is the sum of $M$ noise realizations, whereas it is just a single noise realization in the case of field blending. Note that summing the uncorrelated noise means an increase of $\sqrt{M}$ of the noise power. We illustrate the above theory by a simple numerical example. In Figure 1, computer blending and field blending are simulated for the case that $M=5$ and results are compared. In the case of field blending, one random noise realization was added to the noise-free blended shot record. In the case of blending in the computer, one random noise realization was added to each individual unblended shot record first. All random noise realizations were given the same power and the blending processes in the computer and in the field were exactly the same. The difference in $\mathrm{S} / \mathrm{N}$ can be observed here; see Figure $1 \mathrm{a}$ and $1 \mathrm{~b}$. The noise levels were computed, and, as expected, the results confirmed the theory (reduction by $\sqrt{5}$ ).

\section{S/N IN DEBLENDED DATA}

Now, let us look in the deblended domain and compare the result of a deblending process on field-blended data with unblended field data. Deblending is carried out by minimizing the following expression for all blended shot records (weighted least-squares minimization):

$$
\left(\mathbf{P}_{j}^{\prime}-\sum_{k=1}^{M} \mathbf{P}_{k} \gamma_{k j}\right)^{H} \boldsymbol{\Lambda}\left(\mathbf{P}_{j}^{\prime}-\sum_{k=1}^{M} \mathbf{P}_{k} \gamma_{k j}\right)=\text { minimum. }
$$

Here, $\mathbf{P}_{k}$ is a deblended shot record and $\boldsymbol{\Lambda}$ is a diagonal matrix containing the least-squares weights. In practice, constraints are needed such as causality. In addition, deblending algorithms often include some coherence filtering in the receiver domain (Abma et al., 2012;
Beasley et al., 2012; Doulgeris et al., 2012). The deblending result can be presented by the following expression:

$$
\left\langle\mathbf{P}_{k}\right\rangle=\mathbf{D X S} \mathbf{S}_{k}+\Delta \mathbf{N}_{k}+\delta \mathbf{N}_{k} \text { for } k=1, \ldots, M,
$$

where $\Delta \mathbf{N}_{k}$ equals the background noise and $\delta \mathbf{N}_{k}$ equals the residual blending noise (remaining interference). Bear in mind that after reblending the deblended shot records, the difference with the blended field data is minimum according to equation 5. Typically, we find that the residue is in the order of $-20 \mathrm{~dB}$. This means that we may write within the accuracy of the residue levels,

$$
\mathbf{N}_{j}=\sum_{k=1}^{M} \Delta \mathbf{N}_{k} \gamma_{k j}
$$

From equation 7, we may conclude that the background-related S/N in the deblended field data (equation 6) is $\sqrt{M}$ higher than in the unblended field data (equation 1). As suggested by Beasley et al. (2012), this property could be exploited in marine surveys by extending the weather window in blended acquisition. On the other hand, it is interesting to realize that in the case of calm weather, the source level could be reduced. This means that blending also offers an attractive opening to minimize the negative effects on sea life. For computer-blended data noise, equation 7 needs to be modified to

$$
\sum_{k=1}^{M} \mathbf{N}_{j} \gamma_{k j}=\sum_{k=1}^{M} \Delta \mathbf{N}_{k} \gamma_{k j} \quad \text { for } k=1, \ldots, M,
$$

showing that in computer-blended data, the deblended data have the same background-related $\mathrm{S} / \mathrm{N}$ as the unblended field data.

We illustrate again the theory by the same numerical example: The deblended field records have been compared with the corresponding unblended field results. The difference in $\mathrm{S} / \mathrm{N}$ can be clearly observed in Figure 2a and 2b. The noise levels were computed, confirming that deblending increases the background $\mathrm{S} / \mathrm{N}$ by the blending fold $(\sqrt{5})$. We also show the results for the computerblended data (compare Figure $3 \mathrm{a}$ and $3 \mathrm{~b}$ ). As expected, the $\mathrm{S} / \mathrm{N}$ in deblended and unblended data is the same. a)

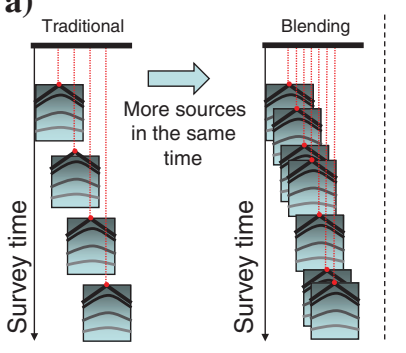

b)

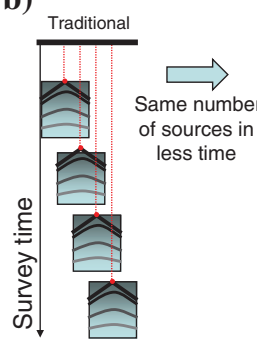

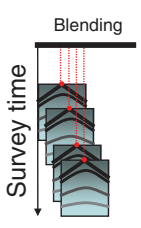

Figure 4. (a) The source power (total signal energy divided by survey time) can be increased by increasing the number of sources while keeping the effective survey time the same. (b) The source power can also be increased by keeping the number of sources equal and making the effective survey time shorter. In both situations, the background-related noise power is the same. 


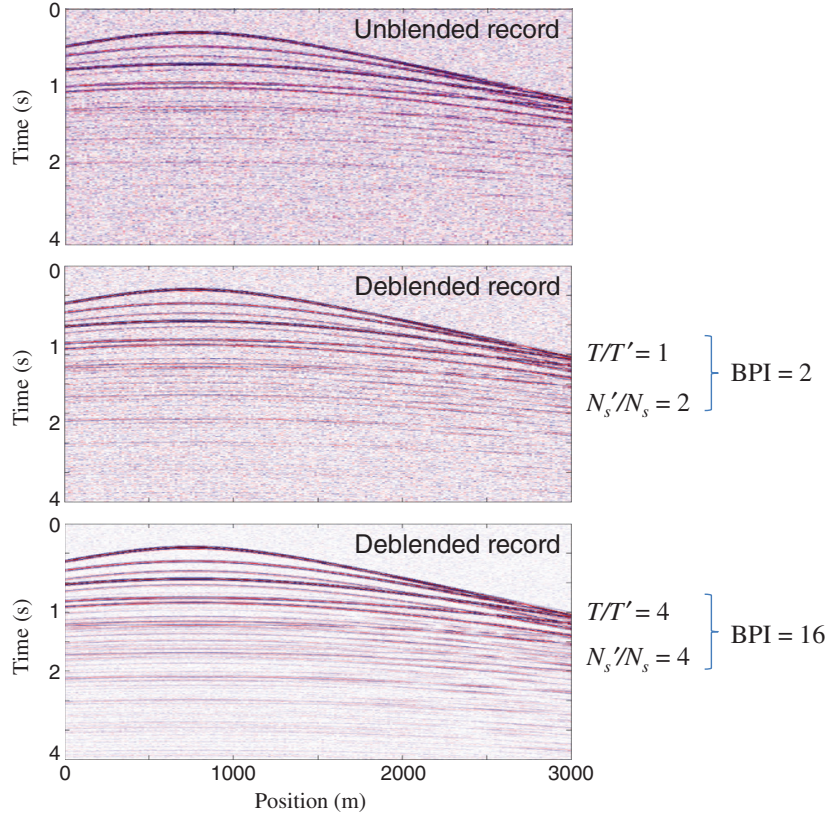

Figure 5. For a given survey time, the background-related $\mathrm{S} / \mathrm{N}$ in the deblended shot records increases with increasing blending fold $\left(\sqrt{N_{s}^{\prime} / N_{s}}\right)$ and with decreasing survey time $\left(\sqrt{T / T^{\prime}}\right)$. In combination, the $\mathrm{S} / \mathrm{N}$ increases linearly with $\sqrt{\mathrm{BPI}}$.

\section{INFLUENCE OF RECORDING TIME}

The improvement in the $\mathrm{S} / \mathrm{N}$ of deblended field data with respect to unblended field data can also be understood by realizing that in blended acquisition, the source wavefield is incoherent. This means that the incoherent source power (total signal energy divided by recording time $T$ ) increases with the square root of the blending fold $(\sqrt{M})$. However, the source power can also be increased by keeping the number of sources equal and making the effective survey time shorter (Figure 4). If we do both, increasing the number of sources and decreasing the effective survey time, then the S/N of the deblended field records increases linearly with $\sqrt{\mathrm{BPI}}$. This important blending and deblending property is illustrated in Figure 5.

\section{CONCLUSIONS}

In field blending, the power of the background noise is independent of the blending process (thus also of the blending fold). In computer blending, the power of the background noise increases with the blending fold.

For a given survey time, the background-related $\mathrm{S} / \mathrm{N}$ in the deblended shot records increases with increasing blending fold $\left(\sqrt{N_{s}^{\prime} / N_{s}}\right)$. Moreover, for a given blending fold, the background-related $\mathrm{S} / \mathrm{N}$ increases with decreasing effective survey time $\left(\sqrt{T / T^{\prime}}\right)$. By combining the two, the background-related $\mathrm{S} / \mathrm{N}$ of deblended field data increases with the square root of the blending performance indicator $(\sqrt{\mathrm{BPI}})$.

Decreasing the effective survey time is not only an economic benefit, it is also a benefit with respect to data quality. The limit in decreasing survey times is given by the incoherency requirement of the blended source wavefield.

By simulating blended field records in the computer (numerical blending), deblending gives an underestimate of the benefit of blending.

Blending is not only an excellent solution for situations with a high background noise (same $\mathrm{S} / \mathrm{N}$ is realized with higher noise levels), it also offers an attractive solution to cope with the increasing legislation on sea life protection (same $\mathrm{S} / \mathrm{N}$ is realized with lower signal levels).

If we analyze blended seismic data after migration, then a second improvement of the $\mathrm{S} / \mathrm{N}$ can be observed due to the improved source sampling (resulting in less aliasing noise). Both properties of field blending, more source power and better source sampling, explain the large increase in image quality that can be seen in practice.

\section{REFERENCES}

Abma, R., Q. Zhang, A. Arogunmati, and G. Beaudoin, 2012, An overview of BP's marine independent simultaneous source field trials: 82nd Annual International Meeting, SEG, Expanded Abstracts, doi: 10.1190/ segam2012-1404.1

Bagaini, C., 2006, Overview of simultaneous vibroseis acquisition methods: 76th Annual International Meeting, SEG, Expanded Abstracts, 70-74.

Beasley, C. J., R. E. Chambers, and Z. Jiang, 1998, A new look at simultaneous sources: 68th Annual International Meeting, SEG, Expanded Abstracts, 133-135.

Beasley, C. J., B. Dragoset, and A. Salama, 2012, A 3D simultaneous source field test processed using alternating projections: A new active separation method: Geophysical Prospecting, 60, 591-601, doi: 10.1111/j.13652478.2011.01038.x.

Berkhout, A., D. Verschuur, and G. Blacquière, 2012, Illumination properties and imaging promises of blended, multiple-scattering seismic data: A tutorial: Geophysical Prospecting, 60, 713-732, doi: 10.1111/j.13652478.2012.01081.x.

Berkhout, A. J., 1982, Seismic migration: Imaging of acoustic energy by wave field extrapolation, A: Theoretical aspects: Elsevier.

Berkhout, A. J., 2008, Changing the mindset in seismic data acquisition: The Leading Edge, 27, 924-938, doi: 10.1190/1.2954035.

Berkhout, A. J., and G. Blacquière, 2012, Utilizing dispersed source arrays in blended acquisition: 82nd Annual International Meeting, SEG, Expanded Abstracts, doi: 10.1190/segam2012-0302.1.

Doulgeris, P., K. Bube, G. Hampson, and G. Blacquière, 2012, Convergence analysis of a coherency-constrained inversion for the separation of blended data: Geophysical Prospecting, 60, 769-781, doi: 10.1111/j .1365-2478.2012.01088.x.

Hampson, G., J. Stefani, and F. Herkenhoff, 2008, Acquisition using simultaneous sources: The Leading Edge, 27, 918-923, doi: 10.1190/1.2954034.

Howe, D., M. Foster, T. Allen, B. Taylor, and I. Jack, 2008, Independent simultaneous sweeping - A method to increase the productivity of land seismic crews: 78th Annual International Meeting, SEG, Expanded Abstracts, 2826-2830.

Ikelle, L., 2007, Coding and decoding: Seismic data modeling, acquisition and processing: 77th Annual International Meeting, SEG, Expanded Abstracts, 66-70.

Krupovnickas, T., K. Matson, C. Corcoran, and R. Pascual, 2012, Marine simultaneous source OBS survey suitability for 4D analysis: 82nd Annual International Meeting, SEG, Expanded Abstracts, doi: 10.1190/ segam2012-0815.1.

Pecholcs, P. I., S. K. Lafon, T. Al-Ghamdi, H. Al-Shammery, P. G. Kelamis, S. X. Huo, O. Winter, J.-B. Kerboul, and T. Klein, 2010, Over 40,000 vibrator points per day with real-time quality control: Opportunities and challenges: 80th Annual International Meeting, SEG, Expanded Abstracts, 111-115.

Stefani, J., G. Hampson, and F. Herkenhoff, 2007, Acquisition using simultaneous sources: 69th Annual International Conference and Exhibition, EAGE, Extended Abstracts, B006.

Vaage, S. T., 2002, Method and system for acquiring marine seismic data using multiple seismic sources: U.S. Patent 6,906,981. 